CORRECTION

https://doi.org/10.1038/s41586-019-0883-2

\title{
Author Correction: Landscape of somatic mutations in 560 breast cancer whole-genome sequences
}

Serena Nik-Zainal, Helen Davies, Johan Staaf, Manasa Ramakrishna, Dominik Glodzik, Xueqing Zou, Inigo Martincorena, Ludmil B. Alexandrov, Sancha Martin, David C. Wedge, Peter Van Loo, Young Seok Ju, Marcel Smid, Arie B. Brinkman, Sandro Morganella, Miriam R. Aure, Ole Christian Lingjærde, Anita Langerød, Markus Ringnér, Sung-Min Ahn, Sandrine Boyault, Jane E. Brock, Annegien Broeks, Adam Butler, Christine Desmedt, Luc Dirix, Serge Dronov, Aquila Fatima, John A. Foekens, Moritz Gerstung, Gerrit K. J. Hooijer, Se Jin Jang, David R. Jones, Hyung-Yong Kim, Tari A. King, Savitri Krishnamurthy, Hee Jin Lee, Jeong-Yeon Lee, Yilong Li, Stuart McLaren, Andrew Menzies, Ville Mustonen, Sarah O'Meara, Iris Pauporté, Xavier Pivot, Colin A. Purdie, Keiran Raine, Kamna Ramakrishnan, F. Germán Rodríguez-González, Gilles Romieu, Anieta M. Sieuwerts, Peter T. Simpson, Rebecca Shepherd, Lucy Stebbings, Olafur A. Stefansson, Jon Teague, Stefania Tommasi, Isabelle Treilleux, Gert G. Van den Eynden, Peter Vermeulen, Anne VincentSalomon, Lucy Yates, Carlos Caldas, Laura van't Veer, Andrew Tutt, Stian Knappskog, Benita Kiat Tee Tan, Jos Jonkers, Åke Borg, Naoto T. Ueno, Christos Sotiriou, Alain Viari, P. Andrew Futreal, Peter J. Campbell, Paul N. Span, Steven Van Laere, Sunil R. Lakhani, Jorunn E. Eyfjord, Alastair M. Thompson, Ewan Birney, Hendrik G. Stunnenberg, Marc J. van de Vijver, John W. M. Martens, Anne-Lise Børresen-Dale, Andrea L. Richardson, Gu Kong, Gilles Thomas \& Michael R. Stratton

Correction to: Nature https://doi.org/10.1038/nature17676, published online 2 May 2016.

In the Methods section of this Article, 'greater than' should have been 'less than' in the sentence 'Putative regions of clustered rearrangements were identified as having an average inter-rearrangement distance that was at least 10 times greater than the whole-genome average for the individual sample.'. The original Article has not been corrected online. 